\title{
Scenario-based Component Behavior Filtration ${ }^{\star}$
}

\author{
Yan Zhang, Xiaofeng Yu, Tian Zhang, Xuandong Li, and Guoliang Zheng \\ State Key Laboratory of Novel Software Technology \\ Department of Computer Science and Technology \\ Nanjing University, Nanjing, P. R. China 210093 \\ zhangyan@seg.nju.edu.cn, Ixd@nju.edu.cn
}

\begin{abstract}
Components with undesired behavior could not be used properly by users. Therefore, the scenario-based behavior filtration of components is a significant problem to be solved, where the scenarios specify what behavior is undesired and what is desired. We propose an approach for filtering out the undesired behavior specified by a scenario specification from components. The main idea of our approach is that by constructing a special environment, i.e., conditional exclusive environment, for a component, all undesired behavior specified by one scenario specification can be filtered out and all desired behavior specified by another scenario specification can be preserved when the component works in the environment. We use interface automata to model the behavior of components and a set of action sequences to abstract the scenario specification in message sequence charts. The composition of components is modelled by the product of interface automata. We give the relevant algorithm in our approach and illustrate it by an example.
\end{abstract}

\section{Introduction}

Component-based software development (CBSD) is a good approach to attain reliable, flexible, extensible and evolvable systems. By the reuse of existing software components and the plug-and-play mechanisms, complex systems can be developed more rapidly and economically. In CBSD, users retrieve desired components from repositories and composite them to build a new system.

When an existing component could not meet the requirement of users exactly, we can compose several available components to perform the given task $[1,2]$. Although components composition can repair inadequate behavior of sole component, it is insufficient to tackle the undesired behavior in available components. The behavior of a component that could obstruct the use of the component in some scenario may be undesired for specific users. Retrieved components with undesired behavior are frequently encountered by users, because users' requirements are various and it is difficult to find an exact match in repositories.

Usually, users give their requirements by a description of scenarios, which is called the scenario specification. The scenario specification can describe either the

\footnotetext{
* This paper is supported by the National Grand Fundamental Research 973 Program of China (No. 2002CB312001), the National Natural Science Foundation of China (No. 60425204, No. 60233020), and by Jiangsu Province Research Foundation (No. BK2004080).
}

Please use the following format when citing this chapter: 
user's desired or undesired behavior of a component when it interacts with other. The scenario-based behavior filtration of a component is to discard the undesired behavior and preserve desired behavior of the component in terms of the scenario specifications given by a user.

In this paper, we propose an approach to filtering the behavior for a component based on scenarios. By constructing an environment (i.e., another component) for a component, filter out all undesired behavior and preserve all desired behavior of the component when the component works in the environment. The undesired and desired behavior of the component are specified by scenario specifications. Interface automata [3] are used to model the behavior of components. Scenarios are specified by message sequence charts (MSCs) [4] and a MSC is abstracted as a set of action sequences further. The composition of components is modelled by the product of interface automata. We extend the concept of environment in the interface automata theory and introduce conditional exclusive environment (CXE). By constructing a CXE $E$ for a given interface automaton $R$ under two known sets $\mathcal{L}^{+}, \mathcal{L}^{-}$of action sequences, make all behavior represented by some element in $\mathcal{L}^{-}$to be discarded in $R \otimes E$. At the same time, all behavior represented by any element in $\mathcal{L}^{+}$, if it is also the behavior of $R$, is preserved in $R \otimes E$.

The remainder of this paper is organized as follows. Section 2 gives a brief introduction on interface automata and message sequence charts. Section 3 introduces some relevant concepts about our proposal. Section 4 describes the approach to scenariobased behavior filtration of components in detail and shows the constructive algorithm of CXE. Finally, in section 5 we discuss the related works and conclude this paper. Additionally, an example is used to illustrate our approach throughout the paper.

\section{Background}

In the section, interface automata and MSCs are introduced briefiy. The most of concepts about interface automata and MSCs refer to [3] and [4] respectively.

\subsection{Interface Automata}

Definition 1 (interface automaton, IA). An interface automaton $P=$ $\left\langle V_{P}, V_{P}^{\text {init }}, \mathcal{A}_{P}^{I}, \mathcal{A}_{P}^{O}, \mathcal{A}_{P}^{H}, \mathcal{T}_{P}\right\rangle$ is a 6-tuple, where

- $V_{P}$ is a finite set of states.

$-V_{P}^{\text {init }} \subseteq V_{P}$ is a set of initial states. If $V_{P}^{\text {init }}=\emptyset$ then $P$ is called empty.

- $\mathcal{A}_{P}^{I}, \mathcal{A}_{P}^{O}$ and $\mathcal{A}_{P}^{H}$ are mutually disjoint sets of input, output and internal actions. $\mathcal{A}_{P}$ denotes the set of all actions, i.e., $\mathcal{A}_{P}=\mathcal{A}_{P}^{I} \cup \mathcal{A}_{P}^{O} \cup \mathcal{A}_{P}^{H}$.

- $\mathcal{T}_{P} \subseteq V_{P} \times \mathcal{A}_{P} \times V_{P}$ is a set of steps. If $\tau=(v, a, u) \in \mathcal{T}_{P}$, then write $\operatorname{label}(\tau)=a, \operatorname{head}(\tau)=v, \operatorname{tail}(\tau)=u$.

If $a \in \mathcal{A}_{P}^{I}$ (resp. $a \in \mathcal{A}_{P}^{O}, a \in \mathcal{A}_{P}^{H}$ ), then $\left(v, a, v^{\prime}\right)$ is called an input (resp. output, internal) step. If there is a step $\left(v, a, v^{\prime}\right) \in \mathcal{T}_{P}$ for some $v, v^{\prime} \in V_{P}, a \in \mathcal{A}_{P}$, then we say that action $a$ is enabled at state $v$. For $v \in V_{P}$, let $\mathcal{A}_{P}^{I}(v)=\left\{a \in \mathcal{A}_{P}^{I} \mid \exists v^{\prime} \in\right.$ 
$\left.V_{P} .\left(v, a, v^{\prime}\right) \in \mathcal{T}_{P}\right\}, \mathcal{A}_{P}^{O}(v)=\left\{a \in \mathcal{A}_{P}^{O} \mid \exists v^{\prime} \in V_{P} .\left(v, a, v^{\prime}\right) \in \mathcal{T}_{P}\right\}$ and $\mathcal{A}_{P}^{H}(v)=$ $\left\{a \in \mathcal{A}_{P}^{H} \mid \exists v^{\prime} \in V_{P} .\left(v, a, v^{\prime}\right) \in \mathcal{T}_{P}\right\}$ be respectively the subset of input, output and internal actions that are enabled at the state $v$. Let $\mathcal{A}_{P}(v)=\mathcal{A}_{P}^{I}(v) \cup \mathcal{A}_{P}^{O}(v) \cup \mathcal{A}_{P}^{H}(v)$.

If IA $P$ satisfies $\left|V_{P}^{\text {init }}\right|=1$ and $\forall(v, a, u),\left(v, a, u^{\prime}\right) \in \mathcal{T}_{P} \cdot u=u^{\prime}$, then $P$ is deterministic, otherwise $P$ is non-deterministic. For simplicity, we make a convention that all interface automata referred in this paper are deterministic.

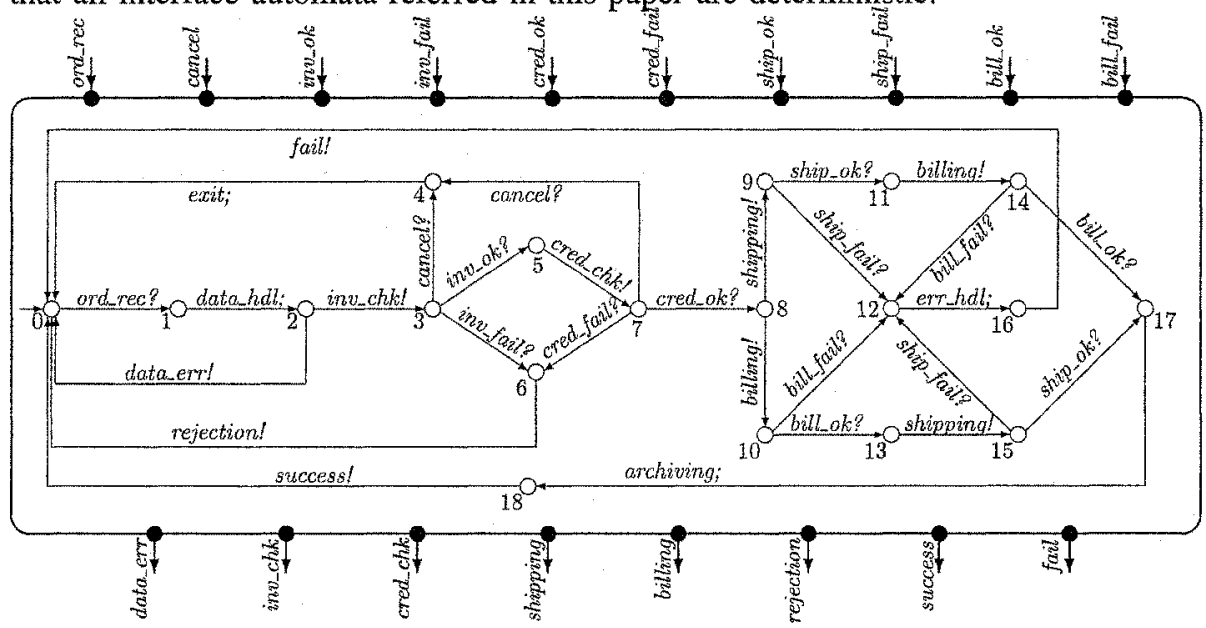

Fig. 1. Interface automaton Seller. The symbol "?" (resp. "!", ";") appended to the name of actions denotes that the action is an input (resp. output, internal) action. An arrow without source denotes the initial state of the interface automaton

Example 1. The IA Seller (see Fig. 1) specifies the behavior of a component when it interacts with other. The component stands for a seller in a business to business system. The seller receives an order (ord_rec) from a customer and handles data in the order (data_hdl), e.g., transform of data format. If there is some error in the order, it will report the error (data_err) to the customer, otherwise it continues to check the inventory $\left(i n v_{-} c h k\right)$ from the supplier and the customer credit (cred_chk) from the bank. Contingent on availability of inventory $\left(i n v_{-} o k\right)$ and valid credit (cred_ok), the seller will inform the shipper to ship product (shipping) and the bank to bill the customer for the order (billing). Either unavailability of inventory (inv_fail) or invalid credit (cred_fail) will lead to reject the order (rejection). The seller can receive some information (cancel) from the customer to terminate (exit) the order. If shipping and billing finish successfully (ship_ok and bill_ok), the seller will make archive (archiving) and give the notification (success) to the customer. Otherwise the negative notification $(f a i l)$ will be given after processing the exception ( $\left.\mathrm{err} \_h d l\right)$.

An execution fragment of IA $P$ is a finite alternating sequence of states and actions $v_{0} a_{0} v_{1} a_{1} \cdots a_{n-1} v_{n}$, where $\left(v_{i}, a_{i}, v_{i+1}\right) \in \mathcal{T}_{P}$, for all $0 \leq i<n$. Given two states $v, u \in V_{P}$, we say that $u$ is reachable from $v$ if there is an execution fragment with $v$ as the first state and $u$ as the last state. The state $u$ is reachable in $P$ if there is an initial state $v \in V_{P}^{i n i t}$ such that $u$ is reachable from $v$. 
Let $\Gamma_{P}$ denote the set of all execution fragments in IA $P$. For every $\eta \in \Gamma_{P}$, write the first state of $\eta$ as $f \operatorname{irst}(\eta)$, the last state of $\eta$ as last $(\eta)$ and the set of all states of $\eta$ as $V(\eta)$.

Definition 2 (interface automata product). Two IAs $P$ and $Q$ are composable if $\mathcal{A}_{P}^{H} \cap \mathcal{A}_{Q}=\emptyset, \mathcal{A}_{Q}^{H} \cap \mathcal{A}_{P}=\emptyset, \mathcal{A}_{P}^{I} \cap \mathcal{A}_{Q}^{I}=\emptyset$ and $\mathcal{A}_{P}^{O} \cap \mathcal{A}_{Q}^{O}=\emptyset$. Let shared $(P, Q)=$ $\mathcal{A}_{P} \cap \mathcal{A}_{Q}=\left(\mathcal{A}_{P}^{I} \cap \mathcal{A}_{Q}^{O}\right) \cup\left(\mathcal{A}_{P}^{O} \cap \mathcal{A}_{Q}^{I}\right)$ be the set of shared actions of $P$ and $Q$. The product of $P$ and $Q$, denoted by $P \otimes Q$, is the $I A$ defined by

$$
\begin{aligned}
V_{P \otimes Q} & =V_{P} \times V_{Q} \\
V_{P \otimes Q}^{\text {init }} & =V_{P}^{\text {init }} \times V_{Q}^{\text {init }} \\
\mathcal{A}_{P \otimes Q}^{I} & =\left(\mathcal{A}_{P}^{I} \cup \mathcal{A}_{Q}^{I}\right) \backslash \operatorname{shared}(P, Q) \\
\mathcal{A}_{P Q Q}^{O} & =\left(\mathcal{A}_{P}^{O} \cup \mathcal{A}_{Q}^{O}\right) \backslash \operatorname{shared}(P, Q) \\
\mathcal{A}_{P \otimes Q}^{H} & =\mathcal{A}_{P}^{H} \cup \mathcal{A}_{Q}^{H} \cup \operatorname{shared}(P, Q) \\
\mathcal{T}_{P \otimes Q} & =\left\{\left((v, u), a,\left(v^{\prime}, u\right)\right) \mid\left(v, a, v^{\prime}\right) \in \mathcal{T}_{P} \wedge a \notin \operatorname{shared}(P, Q) \wedge u \in V_{Q}\right\} \\
& \cup\left\{\left((v, u), a,\left(v, u^{\prime}\right)\right) \mid\left(u, a, u^{\prime}\right) \in \mathcal{T}_{Q} \wedge a \notin \operatorname{shared}(P, Q) \wedge v \in V_{P}\right\} \\
& \cup\left\{\left((v, u), a,\left(v^{\prime}, u^{\prime}\right)\right) \mid\left(v, a, v^{\prime}\right) \in \mathcal{T}_{P} \wedge\left(u, a, u^{\prime}\right) \in \mathcal{T}_{Q} \wedge a \in \operatorname{shared}(P, Q)\right\} .
\end{aligned}
$$

At some state of $P \otimes Q$, one IA, say $P$ (or $Q$ ), may produces an output action that is an input action of $Q$ (or $P$ ), but isn't enabled at the current state in $Q$ (or $P$ ). Such state is an illegal states of $P \otimes Q$. For two composable IAs $P$ and $Q$, the set of illegal states of $P \otimes Q$ is denoted by $\operatorname{Illegal}(P, Q) \subseteq V_{P} \times V_{Q}$,

$$
\begin{aligned}
\operatorname{Illegal}(P, Q)=\{(v, & u) \in V_{P} \times V_{Q} \mid \exists a \in \operatorname{shared}(P, Q) . \\
& \left(\left(a \in \mathcal{A}_{P}^{O}(v) \wedge a \notin \mathcal{A}_{Q}^{I}(u)\right) \vee\left(a \in \mathcal{A}_{Q}^{O}(u) \wedge a \notin \mathcal{A}_{P}^{I}(v)\right)\right\} .
\end{aligned}
$$

Definition 3 (environment). An IA $E$ is an environment for an $I A R$ if: (1) $E$ and $R$ are composable, (2) $E$ is not empty, (3) $\mathcal{A}_{E}^{I}=\mathcal{A}_{R}^{O}$, and (4) if Illegall $(R, E) \neq \emptyset$, then no state in Illegal $(R, E)$ is reachable in $R \otimes E$.

\subsection{Message Sequence Charts}

MSC [4] is a trace description language for visualization of selected system runs. It concentrates on message interchange by communicating entities and their environment. Every MSC specification has an equivalent graphical and textual representation. Especially the graphical representation of MSCs gives an intuitive understanding of the described system behavior. Therefore, MSC is a widely used language for scenario specifications.

The fundamental language constructs of MSCs are component and message flow. Vertical time lines with a named heading represent components. Along these time lines, MSC events (i.e., message send or receive events) are arranged that gives an order to the events connected to this component. A message is depicted by an arrow from the send to the receive event. The fact that a message must be sent before it can be received imposes a total order on the send and receive event of a message and, 
furthermore, a partial order on all events in a MSC. An example of MSCs is shown in Fig. 2 .

Definition 4 (message sequence chart, MSC). A message sequence chart $C h=$ $\langle\mathcal{C}, \mathcal{E}, \mathcal{M}, \mathcal{F}, \mathcal{O}\rangle$ is a 5 -tuple, where

$-\mathcal{C}$ is a finite set of components.

- $\mathcal{E}$ is a finite set of events corresponding to sending or receiving a message.

- $\mathcal{M}$ is a finite set of messages. For any $m \in \mathcal{M}$, let $s(m)$ and $r(m)$ to denote the events that correspond to sending and receiving message $m$ respectively.

$-\mathcal{F}: \mathcal{E} \rightarrow \mathcal{C}$ is a labelling function which maps each event to a component.

- $\mathcal{O} \subseteq \mathcal{E} \times \mathcal{E}$ is a partial order relation over the set of events. For every $\left(e, e^{\prime}\right) \in \mathcal{O}$, there is $e \neq e^{\prime} .\left(e, e^{\prime}\right)$ represents a visual order displayed in $C h$.

Each MSC describes a set of message sequences. A message sequence of one MSC must be composed of all messages of the MSC and any message occurs only once in the sequence. For any two messages in the sequence, if one precedes the other then their send events and receive events should not violate the partial order relation over the set of events. Observe that messages in MSCs correspond to actions in IA. Hence, we call a message sequence of MSC as an action sequence derived from the MSC and write it as $\varrho=\varrho(0) \varrho(1) \cdots \varrho(n)$, where $\varrho(i)$ is a message in the message sequence for all $0 \leq i \leq n$.

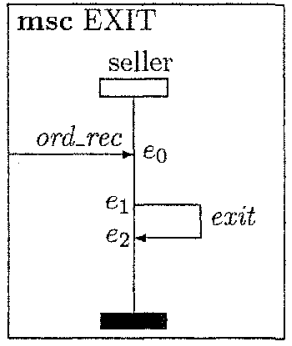

(a) MSC 'EXIT'

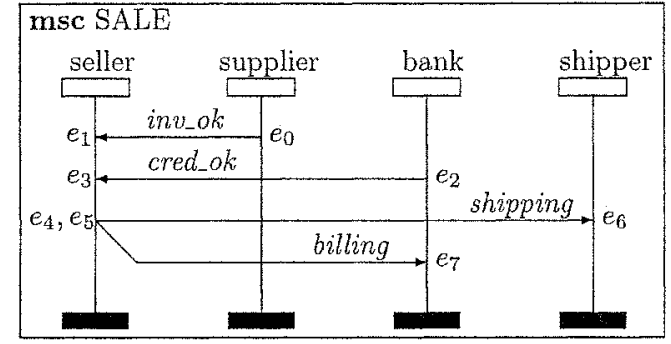

(b) MSC 'SALE'

Fig. 2. MSCs specifying scenarios about the interaction among the seller component, consumers and other components

Example 2. The MSCs 'EXIT' and 'SALE' (see Fig. 2(a) and 2(b) respectively) show two scenario specifications about the seller component (in Example 1) interacting with consumers and other components. The MSC 'EXIT' describes a scenario: the seller interrupts the process of ordering and exits after it receives an order from a customer. From the MSC 'EXIT' we can derive a set of action sequences, $\mathcal{L}_{\mathrm{E}}=\{$ ord_rec exit $\}$. For legibility, we use the symbol "^" to separate two adjacent actions in an action sequence. The MSC 'SALE' describes a scenario: if the seller receives $i n v_{-} o k$ and cred_ok it should produce shipping to the shipper and billing to the bank. From the MSC 'SALE' we can derive a set of action sequences, $\mathcal{L}_{\mathrm{S}}=\left\{i n v_{-} o k^{\wedge} c r e d_{-}\right.$ok^shipping^billing, cred_okinv_ok^shipping

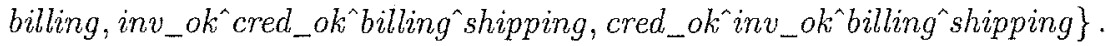




\section{Conditional Exclusive Environment}

For any execution fragment $\eta=v_{i} a_{i} v_{i+1} a_{i+1} \cdots a_{j-1} v_{j}(i<j)$ of IA $P$, where $v_{i} \in V_{P}^{\text {init }}$, if $v_{i}=v_{j}$ or $\mathcal{A}_{P}\left(v_{j}\right)=\emptyset$, then $\eta$ is called a run in $P$. Let $\Sigma_{P}$ denote the set of all runs in IA $P$. For any execution fragment $\eta=v_{i} a_{i} v_{i+1} a_{i+1} \cdots a_{j-1} v_{j} \in \Gamma_{P}$ $(i<j)$, we say that execution fragment $\eta^{\prime}=v_{s} a_{s} v_{s+1} a_{s+1} \cdots a_{t-1} v_{t}(i \leq s<t \leq j)$ is in $\eta$, denoted by $\eta^{\prime} \sqsubseteq \eta$. Specifically, if $\eta^{\prime}=v_{s} a_{s} v_{s+1}(i \leq s<j)$, then we say that the step $\tau=\left(v_{s}, a_{s}, v_{s+1}\right) \in \mathcal{T}_{P}$ is in the execution fragment $\eta$, denoted by $\tau \sqsubseteq \eta$.

The trace of an execution fragment $\eta=v_{0} a_{0} v_{1} a_{1} \cdots a_{n-1} v_{n}$ is a subsequence of $\eta$, which consists of all actions in $\eta$. We write $\operatorname{trace}(\eta)=a_{0} a_{1} \cdots a_{n-1}$. Given an execution fragment $\eta \in \Gamma_{P \otimes Q}$ and $\operatorname{trace}(\eta)=a_{0} a_{1} \cdots a_{n-1}$, the projection of $\eta$ on IA $P$, denoted by $\pi_{P}(\operatorname{trace}(\eta))$, is a subsequence of trace $(\eta)$, which is obtained by deleting all actions $a_{i} \in \mathcal{A}_{Q} \backslash \operatorname{shared}(P, Q), 0 \leq i \leq n-1$ from $\operatorname{trace}(\eta)$.

Given two composable IAs $P$ and $Q$, there are $\eta=v_{0} a_{0} v_{1} a_{1} \cdots a_{n-1} v_{n} \in \Gamma_{P}$ and $\alpha \in \Sigma_{P \otimes Q}$. If there exists an execution fragment $\zeta \sqsubseteq \alpha$ satisfying $\pi_{P}(\operatorname{trace}(\zeta))=$ trace $(\eta)$ and for any $v_{i} a_{i} v_{i+1} \sqsubseteq \eta$ there is $\left(v_{i}, u_{i}\right) a_{i}\left(v_{i+1}, u_{i+1}\right) \sqsubseteq \zeta$, where $u_{i}, u_{i+1} \in V_{Q}$ and $0 \leq i<n$, then we say that $\eta$ is covered by $\alpha$. At the same time, $\left(u_{i}, a_{i}, u_{i+1}\right)$ is called the corresponding step of $\left(v_{i}, a_{i}, v_{i+1}\right)$ if $a_{i} \in \operatorname{shared}(P, Q)$, and $u_{i}, u_{i+1}$ is called the corresponding state of $v_{i}, v_{i+1}$ respectively. If an execution fragment of IA $P$ can be covered by a run of IA $P \otimes Q$, then it means that the behavior represented by the execution fragment of $P$ can be preserved in $P \otimes Q$.

Given a run $\alpha$ of IA $P$ and an action sequence $\varrho$, if $\varrho$ is a subsequence of $\operatorname{trace}(\alpha)$, then we say action sequence $\varrho$ occurs in run $\alpha$, denoted by $\varrho \propto \alpha$. The occurrence of an action sequence in a run of one IA means that some behavior of the IA contains the behavior represented by the action sequence.

Suppose that action sequence $\varrho=\varrho(0) \varrho(1) \cdots \varrho(m)$ occurs in run $\alpha \in \Sigma_{P}$. If there exists an execution fragment $\eta \sqsubseteq \alpha$ satisfying that $\varrho$ is a subsequence of trace $(\eta)=a_{0} a_{1} \cdots a_{n}(n \geq m)$ and $\varrho(0)=a_{0}, \varrho(m)=a_{n}$, then $\eta$ is a proper occurrence of $\varrho$ in $\alpha$. Suppose that $\eta_{0}, \eta_{1}, \ldots, \eta_{n} \subseteq \alpha$ are the proper occurrences of action sequences $\varrho_{0}, \varrho_{1}, \ldots, \varrho_{n}$ in $\alpha$ respectively. For any $\eta \sqsubseteq \alpha$, if $(V(\eta)$ $\{$ first $(\eta)$, last $(\eta)\}) \cap V\left(\eta_{i}\right)=\emptyset, i=0,1, \ldots, n$, then $\eta$ is a proper inoccurrence of $\varrho_{0}, \varrho_{1}, \ldots, \varrho_{n}$ in $\alpha$.

Given a set $\mathcal{L}$ of action sequences, for any IA $P, \Sigma_{P}$ can be partitioned as two subsets: $\phi_{\mathcal{L}}\left(\Sigma_{P}\right)=\left\{\alpha \in \Sigma_{P} \mid \exists \varrho \in \mathcal{L} . \varrho \propto \alpha\right\}$ and $\overline{\phi_{\mathcal{L}}\left(\Sigma_{P}\right)}=\Sigma_{P} \backslash \phi_{\mathcal{L}}\left(\Sigma_{P}\right)$. For every run in $\phi_{\mathcal{L}}\left(\Sigma_{P}\right)$, there exists at least one action sequence in $\mathcal{L}$ that occurs in it. For any run in $\overline{\phi_{\mathcal{L}}\left(\Sigma_{P}\right)}$, no action sequence in $\mathcal{L}$ occurs in it.

Definition 5 (conditional exclusive environment, CXE). Given an IA $R$ and a set $\mathcal{L}^{-}$of action sequences, the exclusive environment of $R$ under $\mathcal{L}^{-}$is an environment $E$ of $R$ such that for any $\varrho \in \mathcal{L}^{-}$, if $\varrho$ occurs in a run $\alpha$ of $R$, then the proper occurrence of $\varrho$ in $\alpha$ must be not covered by any run of $R \otimes E$. If an exclusive environment $E$ of $R$ under $\mathcal{L}^{-}$satisfies that for any $\varrho \in \mathcal{L}^{+}$, if $\varrho$ occurs in a run $\alpha$ of $R$ then $\alpha$ must be covered by some run of $R \otimes E$, where $\mathcal{L}^{+}$is a set of action sequences and $\mathcal{L}^{+} \cap \mathcal{L}^{-}=\emptyset$, then $E$ is a conditional exclusive environment of $R$ under exclusion condition $\mathcal{L}^{-}$and inclusion condition $\mathcal{L}^{+}$. 
Let $C X E\left(R: \mathcal{L}^{-}, \mathcal{L}^{+}\right)$denote the set of conditional exclusive environments of $R$ under exclusion condition $\mathcal{L}^{-}$and inclusion condition $\mathcal{L}^{+}$. If we consider $\mathcal{L}^{+}$and $\mathcal{L}^{-}$ as the representation of two sets of behavior, then all behavior of $R$ which contain any behavior in $\mathcal{L}^{-}$isn't preserved in $R \otimes E$, at the same time, all behavior of $R$ which contain any behavior in $\mathcal{L}^{+}$is preserved in $R \otimes E$, where $E \in C X E\left(R: \mathcal{L}^{-}, \mathcal{L}^{+}\right)$. For arbitrary IA $R$ and two sets $\mathcal{L}^{-}, \mathcal{L}^{+}$of action sequences, it is possible that $C X E\left(R: \mathcal{L}^{-}, \mathcal{L}^{+}\right)=\emptyset$. It means that a CXE of $R$ under exclusion condition $\mathcal{L}^{-}$ and inclusion condition $\mathcal{L}^{+}$may not always exist.

\section{Construction of Conditional Exclusive Environment}

We can use an IA, say $R$, to specify the behavior of a component, say COMP. An user can give his or her undesired and desired behavior about COMP by two scenario specifications in $\mathrm{MSC}$, say ' $\mathrm{SCENE}$ ', and ' $\mathrm{SCENE}^{+}$' respectively. Filtering out the user's undesired behavior from COMP and preserving the desired behavior amounts to constructing a CXE for $R$ under exclusion condition $\mathcal{L}^{-}$and inclusion condition $\mathcal{L}^{+}$, where $\mathcal{L}^{-}, \mathcal{L}^{+}$are the sets of action sequences derived from MSCs 'SCENE ${ }^{-}$, 'SCENE' ${ }^{+}$' respectively. If there exists $E \in C X E\left(R: \mathcal{L}^{-}, \mathcal{L}^{+}\right)$and we can construct it, then all of the user's undesired behavior in $R$ do not exist in $R \otimes E$, at the same time, all of the user's desired behavior in $R$ are preserved in $R \otimes E$.

In this section, we will discuss how to construct a CXE $E \in C X E\left(R: \mathcal{L}^{-}, \mathcal{L}^{+}\right)$ for known IA $R$ and two sets $\mathcal{L}^{-}, \mathcal{L}^{+}$of action sequences in detail, and give the algorithm for constructing $\mathrm{CXE}$.

\subsection{Basic Approach to Constructing CXE}

An environment of one IA, say $R$, can affect the runs of $R$ only by the input actions of $R$. For arbitrary input step $\tau$ on arbitrary run of $R$, if the label of $\tau$ is a shared action of $R$ and its environment and the environment does not provide the input action for $R$ when $R$ needs it, then $R$ cannot go on along the run. For example, if the environment does not provide input action cancel for IA Seller (see Fig. 1) when Seller stays at state 3 , then Seller cannot run along execution fragment " 3 cancel 4 exit 0 " back to initial state. That the environment does not provide input action $\operatorname{label}(\tau)$ for $R$, when $R$ needs it, amounts to no corresponding step of $\tau$ in the environment.

Suppose that $\eta$ is a proper occurrence of some action sequence in $\mathcal{L}^{-}$. Only by not constructing the corresponding step in $E$ for any input step $\tau$ of $R$, where first $(\eta)$ is reachable from $\operatorname{tail}(\tau)$, the CXE $E$ can make $\eta$ not to be covered by any run of $R \otimes E$. For ensuring all runs in $\phi_{\mathcal{L}^{+}}\left(\Sigma_{R}\right)$ to be covered by runs of $R \otimes E$, the input step $\tau$ should not be in any run in $\phi_{\mathcal{L}^{+}}\left(\Sigma_{R}\right)$. We can find all such input steps in $R$ by traversing all runs in $\phi_{\mathcal{L}-}\left(\Sigma_{R}\right)$. But, if there exists a loop (i.e., execution fragment $\eta$ with $\operatorname{first}(\eta)=$ last $(\eta)$ ) in some run, then $\Sigma_{R}$ is an infinite set and the lengths of some runs in $\Sigma_{R}$, i.e., the number of steps in a run, may be also infinite. Accordingly, $\phi_{\mathcal{L}^{-}}\left(\Sigma_{R}\right), \phi_{\mathcal{L}^{+}}\left(\Sigma_{R}\right)$ and the lengths of some runs in them may be infinite. Thus, it is unfeasible to traverse all runs in $\phi_{\mathcal{L}-}\left(\Sigma_{R}\right)$ directly. For getting a feasible approach, we introduce the concepts of the simple run and simple loop. 
Given an IA $R$ and a set $\mathcal{L}$ of action sequences, a run $\alpha=v_{0} a_{0} v_{1} a_{1} \cdots a_{n-1} v_{n}$ of $R$ is a simple run when it satisfies the following conditions:

1. if $\alpha \in \overline{\phi_{\mathcal{L}}\left(\Sigma_{R}\right)}$, then there is $v_{i} \neq v_{j}(0<i<n, 0<j<n, i \neq j)$;

2. if $\alpha \in \phi_{\mathcal{L}}\left(\Sigma_{R}\right)$, then (a) for any proper inoccurrence $\eta=v_{i} a_{i} v_{i+1} \cdots a_{j-1} v_{j}$ $(0 \leq i<j \leq n)$ in $\alpha$, there is $v_{s} \neq v_{t}(i \leq s \leq j, i \leq t \leq j, s \neq t)$; and (b) for any proper occurrence $\zeta$ of $\varrho=\varrho(0) \varrho(1) \cdots \varrho(m) \in \mathcal{L}$ in $\alpha$, if there is $\zeta^{\prime}=v_{i} a_{i} v_{i+1} a_{i+1} \cdots a_{j-1} v_{j} \sqsubseteq \zeta(0 \leq i<j \leq n)$, and $a_{i}=\varrho(k)$, $a_{j-1}=\varrho(k+1), 0 \leq k<m$, then there is $v_{s} \neq v_{t}(i<s<j, i<t<j, s \neq t)$.

We put some constrains on runs to get the definition of the simple run. The meaning of the condition 1 . is that there is not any loop in a simple run without occurrence of action sequences in $\mathcal{L}$. The meaning of the condition $2 \mathrm{a}$ is that there is not any loop in a proper inoccurrence of action sequences in a simple run. The meaning of the condition $2 b$ is that in a proper occurrence of an action sequence in $a$ simple run, there is not any loop between the occurrence of two neighbor actions in the action sequence. The set of all simple runs of IA $R$ under $\mathcal{L}$ is denoted by $\Omega_{R}^{\mathcal{L}}$. Similarly, $\Omega_{R}^{\mathcal{L}}$ can be partitioned as $\phi_{\mathcal{L}}\left(\Omega_{R}^{\mathcal{L}}\right)$ and $\overline{\phi_{\mathcal{L}}\left(\Omega_{R}^{\mathcal{L}}\right)}$.

Given an IA $R$ and a set $\mathcal{L}$ of action sequences, an execution fragment $\eta=$ $v_{i} a_{i} v_{i+1} a_{i+1} \cdots a_{j-1} v_{j} \in \Gamma_{R}(i<j)$ is a simple loop if : (1) $v_{i}=v_{j}, v_{i}, v_{j} \notin V_{R}^{\text {init }}$, (2) $v_{s} \neq v_{t}(i \leq s<j, i \leq t<j, s \neq t)$ and (3) $\forall \alpha \in \phi_{\mathcal{L}}\left(\Omega_{R}^{\mathcal{L}}\right) \cdot \eta \nsubseteq \alpha$.

The first and second conditions ensure that except the first and the last states, there aren't duplicate states in a simple loop. The third condition ensures that a simple loop isn't the loop in a proper occurrence of some action sequence in $\mathcal{L}$. For given IA $R$ and set $\mathcal{L}$ of action sequences, $\Theta_{R}^{\mathcal{L}}$ denotes the set of all simple loops of $R$. We say that simple loop $\eta \in \Theta_{R}^{\mathcal{L}}$ associates with simple run $\alpha \in \Omega_{R}^{\mathcal{L}}$ if $V(\eta) \cap V(\alpha) \neq \emptyset$ or $V(\eta) \cap V\left(\eta^{\prime}\right) \neq \emptyset$, where $\eta^{\prime} \in \Theta_{R}^{\mathcal{L}}$ associates with $\alpha$. Let $\psi_{\mathcal{L}}\left(\Theta_{R}^{\mathcal{L}}\right)=\left\{\eta \in \Theta_{\widetilde{R}}^{\mathcal{L}} \mid \exists \alpha \in \phi_{\mathcal{L}}\left(\Omega_{R}^{\mathcal{L}}\right) . \eta\right.$ associates with $\left.\alpha\right\}$ be the set of all simple loops associated with simple runs in $\phi_{\mathcal{L}}\left(\Omega_{R}^{\mathcal{L}}\right)$.

Notice that every step in any run in $\Sigma_{R}$ corresponds to a step in some simple run in $\Omega_{R}^{\mathcal{L}}$ or in some simple loop in $\Theta_{R}^{\mathcal{L}}$. However, $\Omega_{R}^{\mathcal{L}}$ and $\Theta_{R}^{\mathcal{L}}$ are finite sets and the lengths of all simple runs and simple loops are finite. Furthermore, $\phi_{\mathcal{L}}\left(\Omega_{\mathcal{R}}^{\mathcal{L}}\right)$ and $\psi_{\mathcal{L}}\left(\Theta_{R}^{\mathcal{L}}\right)$ are finite sets.

Additionally, we also notice that it is impossible to eliminate the undesired behavior represented by $\varrho \in \mathcal{L}^{-}$from $R \otimes E$ by not constructing the corresponding step in $E$ for any step in $R$ "after" the proper occurrence of $\varrho$. A step $\tau$ "after" a proper occurrence $\eta$ means that $h e a d(\tau)=$ last $(\eta)$ or head $(\tau)$ is reachable from last $(\eta)$.

Suppose that $\zeta$ is a proper occurrence of $\varrho \in \mathcal{L}^{-}$in a simple run $\alpha$ of IA $R$. We call a prefix $\eta$ of $\alpha$ as the minimal simple prefix about $\zeta$ if $\zeta$ is a suffix of $\eta$, where a prefix of $\alpha$ is an execution fragment $\eta$ in $\alpha$ and first $(\eta)=$ first $(\alpha)$; a suffix of $\alpha$ is an execution fragment $\eta$ in $\alpha$ and last $(\eta)=\operatorname{last}(\alpha)$. Let $\lambda_{\mathcal{L}^{-}}\left(\Omega_{R}^{\mathcal{C}^{-}}\right)$denote the set of all minimal simple prefixes about all proper occurrences of any action sequence in $\mathcal{L}^{-}$in any simple run of $R$, i.e.,

$\lambda_{\mathcal{L}^{-}}\left(\Omega_{R}^{\mathcal{L}^{-}}\right)=\left\{\eta \mid \exists \varrho \in \mathcal{L}^{-} \cdot \exists \alpha \in \Omega_{R}^{\mathcal{L}^{-}} \cdot(\zeta\right.$ is a proper occurrence of $\varrho$ in $\alpha) \wedge$

( $\eta$ is the minimal simple prefixabout $\zeta$ in $\alpha$ ) $\}$. 
For any $\eta \in \lambda_{\mathcal{L}^{-}}\left(\Omega_{R}^{\mathcal{C}^{-}}\right)$, there must be a proper occurrence of some $\varrho \in \mathcal{L}^{-}$in $\eta$, and there is not any step "after" the proper occurrence in $\eta$.

Theorem 1. For arbitrary IA $R$ and sets $\mathcal{L}^{-}, \mathcal{L}^{+}$of action sequences, if there exist $\eta \in \lambda_{\mathcal{L}^{-}}\left(\Omega_{R}^{\mathcal{L}^{-}}\right)$such that $\forall \tau \sqsubseteq \eta \cdot \operatorname{label}(\tau) \notin \mathcal{A}_{R}^{I}$, then $C X E\left(R: \mathcal{L}^{-}, \mathcal{L}^{+}\right)=\emptyset$.

In [5], we prove that there maybe exist some kind of execution fragments in one IA, say $P$, which cannot be covered by any run of $P \otimes E$, for any environment $E$ of $P$. Accordingly, we have the theorem as follows.

Theorem 2. For arbitrary IA $R$ and sets $\mathcal{L}^{-}, \mathcal{L}^{+}$of action sequences, there does not exist any $E \in C X E\left(R: \mathcal{L}^{-}, \mathcal{L}^{+}\right)$if there are $\eta_{1}, \eta_{2} \in \Gamma_{R}, \eta_{1} \sqsubseteq \alpha$ and $\eta_{2} \sqsubseteq \beta$, for some $\alpha \in\left(\Omega_{R}^{\mathcal{L}^{+}} \cup \Theta_{R}^{\mathcal{L}^{+}}\right)$and $\beta \in\left(\phi_{\mathcal{C}^{+}}\left(\Omega_{R}^{\mathcal{C}^{+}}\right) \cup \psi_{\mathcal{L}^{+}}\left(\Theta_{R}^{\mathcal{L}^{+}}\right)\right)$, which satisfy any of the following conditions: (1) $\eta_{1}=v_{i} a v_{j}$ and $\eta_{2}=v_{j} b v_{k}$, where $i \neq j \neq k$, $a \notin \operatorname{shared}(R, E), b \in \mathcal{A}_{R}^{I} \cap \operatorname{shared}(R, E)$ and $b \notin \mathcal{A}_{R}\left(v_{i}\right)$. (2) $\eta_{1}=v_{i} a v_{j}$ and $\eta_{2}=v_{i} b v_{k}$, where $i \neq j \neq k, a \notin \operatorname{shared}(R, E), b \in \mathcal{A}_{R}^{I} \cap \operatorname{shared}(R, E)$ and $b \notin \mathcal{A}_{R}\left(v_{j}\right)$. (3) $\eta_{1}=v_{i} a_{i} v_{i+1} a_{i+1} \cdots a_{j-1} v_{j}$ and $\eta_{2}=v_{i} b v_{i}^{\prime}$, where $i<j$, $v_{i}^{\prime} \notin V\left(\eta_{1}\right), a_{k} \notin \operatorname{shared}(R, E), k=i, i+1, \ldots, j-1, b \in \mathcal{A}_{R}^{I} \cap$ shared $(R, E)$ and $\exists v \in V\left(\eta_{1}\right), b \notin \mathcal{A}_{R}(v)$.

\subsection{Algorithm of Constructing CXE}

The skeleton of the constructive algorithm for CXE is described as follows. Step one, for every minimal simple prefix about the proper occurrence of any action sequence in $\mathcal{L}^{-}$in some simple run of $R$, traverse it from the first state and find the first input step in it, which is not in any simple run with occurrence of action sequences in $\mathcal{L}^{+}$ or any simple loop associated with it. Step two, remove these input steps from $R$ and all unreachable states after the removal. Step three, construct corresponding steps in one IA for all residual steps in $R$.

Make the convention of $\mathcal{A}_{E}^{H}=\emptyset$ and $\mathcal{A}_{E}^{O}=\mathcal{A}_{R}^{I}$ [5]. Let $R \downarrow \mathcal{T}$ to denote the IA obtained by removing all steps in $\mathcal{T} \subset \mathcal{T}_{R}$ from $R$ and all unreachable states in $R$ after the removal. The algorithm of constructing CXE $E \in C X E\left(R: \mathcal{L}^{-}, \mathcal{L}^{+}\right)$is shown in Algorithm 1.

We can prove that the return (in line 24) of Algorithm 1 is a CXE of $R$ under exclusion condition $\mathcal{L}^{-}$and inclusion condition $\mathcal{L}^{+}$since it is consistent to Definition 5 . Thus, Algorithm 1 is correct.

About line 1 in Algorithm 1, we had given an algorithm to find which simple run in an IA has the occurrence of a given action sequence in [6] and we can obtain those sets in line 1 based on the algorithm. About line 22 in Algorithm 1, we had given a method of constructing corresponding steps in [5] .

Suppose that the maximal length of all elements in the set $\Omega_{R}^{\mathcal{C}^{-}} \cup \Omega_{R}^{\mathcal{C}^{+}} \cup \Theta_{R}^{\mathcal{C}^{+}}$is $n=\max \left\{\right.$ length $\left.(\eta) \mid \eta \in\left(\Omega_{R}^{\mathcal{L}^{-}} \cup \Omega_{R}^{\mathcal{L}^{+}} \cup \Theta_{R}^{\mathcal{L}^{+}}\right)\right\}$, where length $(\eta)$ is the number of steps in $\eta$. Suppose that $m_{1}=\left|\phi_{\mathcal{L}^{-}}\left(\Omega_{R}^{\mathcal{L}^{-}}\right)\right|, m_{2}=\left|\phi_{\mathcal{L}^{+}}\left(\Omega_{\mathcal{L}^{+}}^{\mathcal{L}^{+}}\right)\right|$are the number 


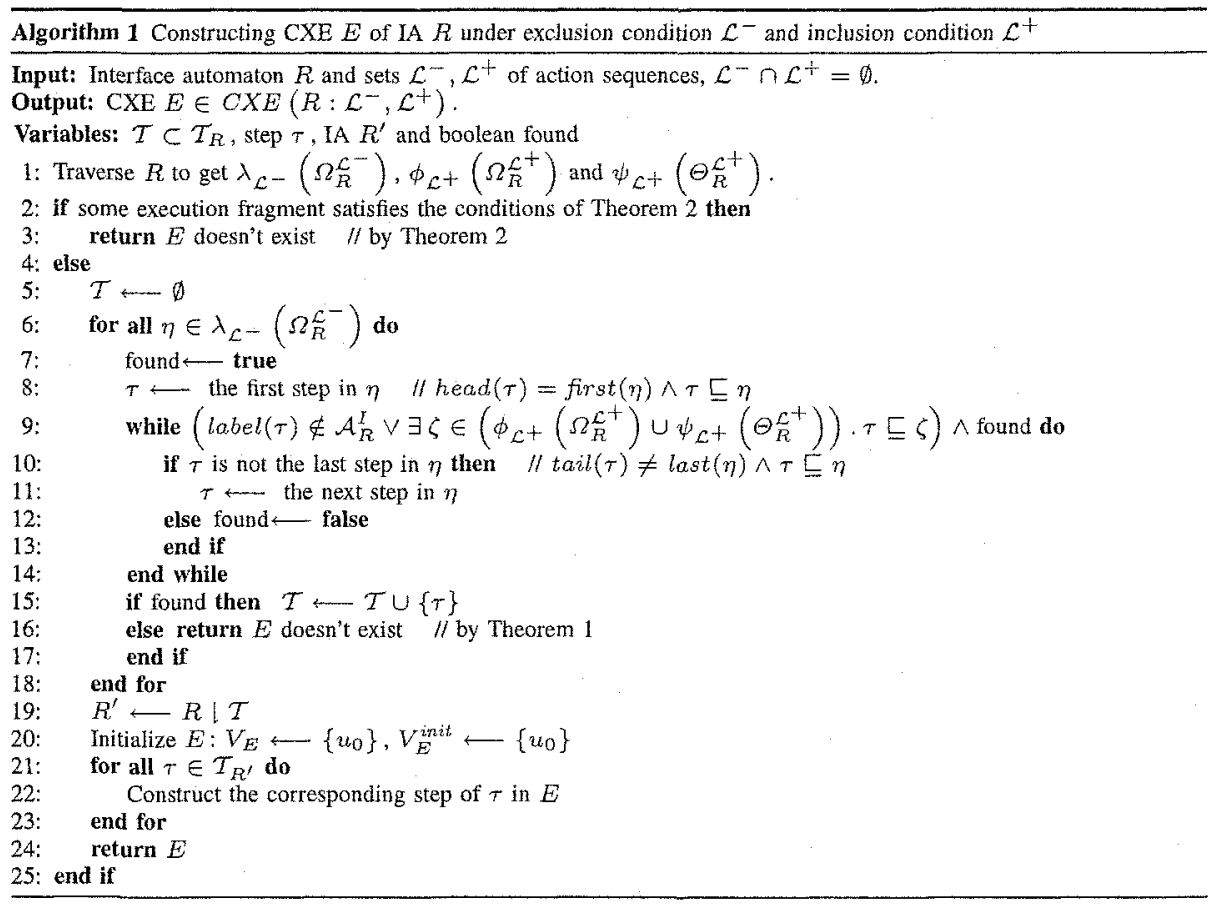

of simple runs in $\phi_{\mathcal{L}^{-}}\left(\Omega_{R}^{\mathcal{C}^{-}}\right), \phi_{\mathcal{L}^{+}}\left(\Omega_{R}^{\mathcal{C}^{+}}\right)$respectively, and $k=\left|\psi_{\mathcal{L}^{+}}\left(\Theta_{R}^{\mathcal{C}^{+}}\right)\right|$ are the number of simple loops in $\psi_{\mathcal{L}^{+}}\left(\Theta_{R}^{\mathcal{L}^{+}}\right)$. In the worst case, line 6 to 18 in Algorithm 1 can be done in $O\left(\left(m_{2}+k\right) m_{1} n^{2}\right)$ time. According to [6] and [5], line 1 and line 22 in Algorithm 1 need $O\left(\left(m_{1}+m_{2}\right) n\right)$ and $O\left(\left|V_{R^{\prime}}\right|\right)$ time respectively, where $\left|V_{R^{\prime}}\right|$ is the number of states of IA $R^{\prime}$. In general, there are length $(\eta) \ll$ length $(\alpha)$ for $\eta \in \Theta_{R}^{\mathcal{L}^{+}}$and $\alpha \in \Omega_{R}^{\mathcal{L}^{+}}$and $\left|V_{R^{\prime}}\right| \ll\left(m_{1}+m_{2}\right) n$. Hence, the complexity of Algorithm 1 is $O\left(m_{1} m_{2} n^{2}\right)$.

Example 3. Suppose that MSCs 'EXIT' (Fig. 2(a)) and 'SALE' (Fig. 2(b)) describe a user's undesired and desired behavior about IA Seller (Fig. 1) respectively. That is, the user does not want the process of ordering to be terminated by cancellation. By Algorithm 1, we can obtain a CXE E (Fig. 4) of the IA Seller under exclusion condition $\mathcal{L}_{\mathrm{E}}$ and inclusion condition $\mathcal{L}_{\mathrm{S}}$, which are two sets of action sequences derived from MSCs 'EXIT' and 'SALE' respectively (see Example 2). The intermediate result $R^{\prime}$ (see line 19 of Algorithm 1) is shown in Fig. 3. It can be found that the user's undesired behavior of Seller is discarded in the composition of Seller and $E$, i.e., Seller $\otimes E$ (Fig. 5). At the same time, the user's desired behavior of Seller is preserved in Seller $\otimes E$.

\section{Related Works and Conclusion}

In this paper, we give an approach for filtering the undesired behavior and preserving the desired behavior of components based on scenario specifications. 


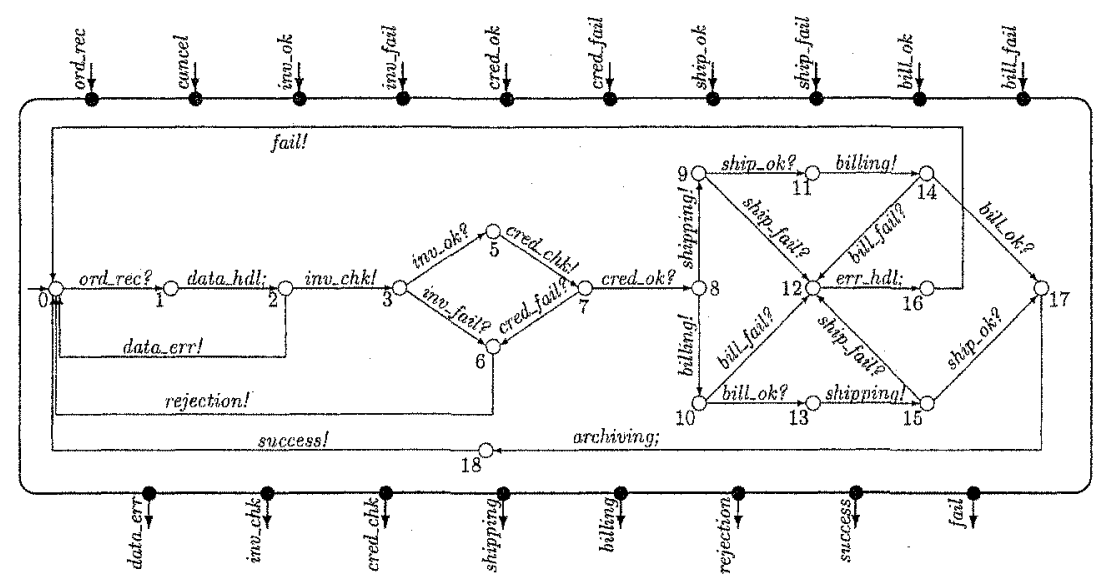

Fig. 3. IA $R^{\prime}$. The intermediate result of Algorithm 1 with inputs Seller, $\mathcal{L}_{\mathrm{E}}$ and $\mathcal{L}_{\mathrm{S}}$

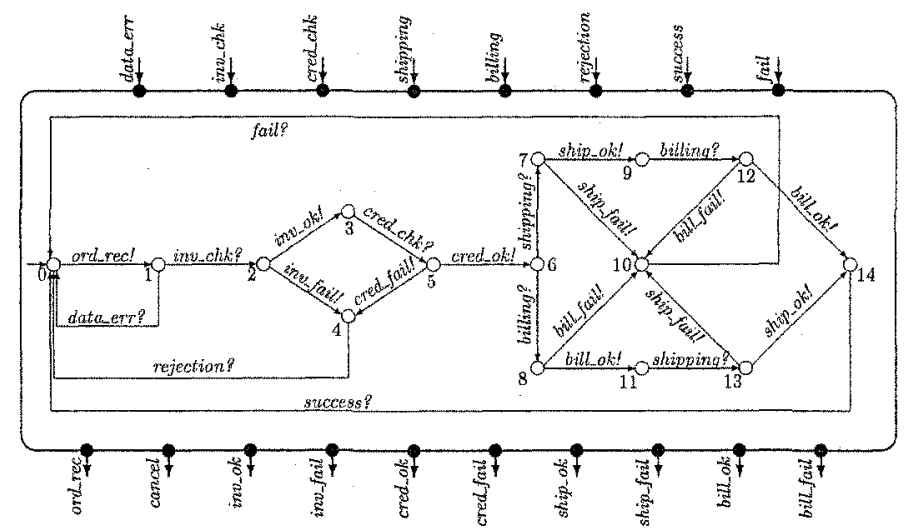

Fig. 4. The CXE $E$ of Seller under exclusion condition $\mathcal{L}_{\mathrm{E}}$ and inclusion condition $\mathcal{L}_{\mathrm{S}}$

In $[1,2,7]$, the authors mainly solve the behavioral compatibility of components composition, but do not concern whether all behavior of the composition are the needs of users. By using environment, our approach can filter out undesired behavior from

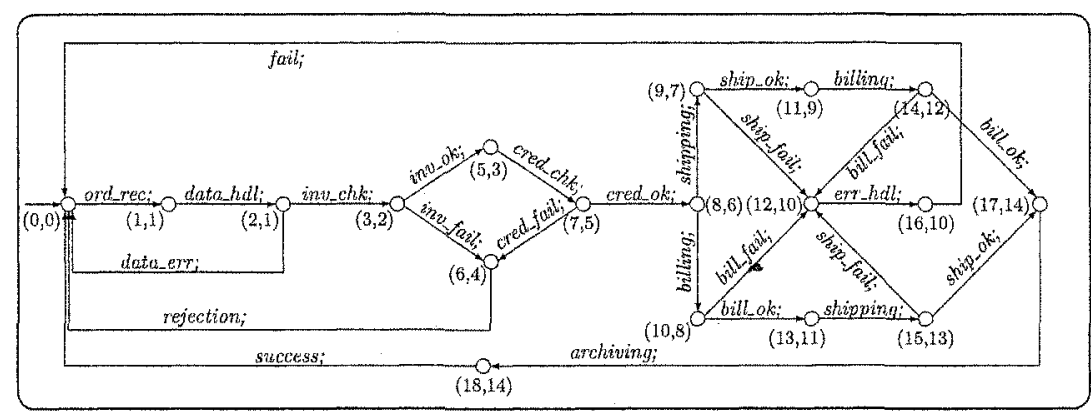

Fig. 5. Seller $\otimes E$. The composition of Seller and $E$ 
components or compositions in terms of the user's requirements. The most pertinent research is to automatically synthesize a connector for restricting the behavior of the composed components to the desired behavior specified by temporal logic based specifications $[8,9]$. Contrary to $[8,9]$, the environment in our approach adjusts the behavior of components only by the inputs, and our algorithm is better in complexity.

\section{References}

1. Bracciali, A., Brogi, A., Canal, C.: A formal approach to component adaptation. Journal of Systems and Software 74(1) (2004) 45-54

2. Yellin, D.M., Strom, R.E.: Protocol specifications and component adaptors. ACM Transactions on Programming Languages and Systems 19(2) (1997) 292-333

3. de Alfaro, L., Henzinger, T.A.: Interface automata. In: Proceedings of the 9th Annual ACM Symposium on Foundations of Software Engineering (FSE 2001), ACM Press (2001) 109-120

4. ITU-TS: ITU-TS recommendation Z.120: Message Sequence Chart (MSC). ITU-TS, Geneva (1999)

5. Zhang, Y., Hu, J., Yu, X., Zhang, T., Li, X., Zheng, G.: Deriving available behavior all out from incompatible component compositions. In: Proceedings of the 2nd International Workshop on Formal Aspects of Component Software (FACS'05), Electronic Notes in Theoretical Computer Science (2006) (To appear).

6. Hu, J., Yu, X., Zhang, Y., Zhang, T., Wang, L., Li, X., Zheng, G.: Scenario-based verification for component-based embedded software designs. In: Proceedings of the 34th International Conference on Parallel Processing Workshops (ICPP 2005 Workshop), IEEE Computer Society (2005) 240-247

7. Schmidt, H.W., Reussner, R.: Generating adapters for concurrent component protocol synchronisation. In: IFIP TC6/WG6.1 Fifth International Conference on Formal Methods for Open Object-Based Distributed Systems, Kluwer (2002) 213-229

8. Inverardi, P., Tivoli, M.: Software architecture for correct components assembly. In Bernardo, M., Inverardi, P., eds.: Formal Methods for Software Architectures. Volume 2804 of Lecture Notes in Computer Science. Springer-Verlag (2003) 92-121

9. Tivoli, M., Autili, M.: SYNTHESIS: a tool for synthesizing "correct" and protocol-enhanced adaptors. L'Object Journal 12(1) (2005) 